\title{
Captures
}

Figures, théories et pratiques de l'imaginaire

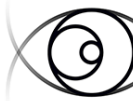

C A P T U R E S Figures, théories et pratiques de limaginaire revue interdisciplinaire

\section{Paroles diffamantes, images infamantes}

\section{Ania Wroblewski}

Volume 4, Number 1, May 2019

Paroles diffamantes, images infamantes

URI: https://id.erudit.org/iderudit/1060152ar

DOI: https://doi.org/10.7202/1060152ar

See table of contents

Publisher(s)

Figura, Centre de recherche sur le texte et l'imaginaire

ISSN

2371-1930 (digital)

Explore this journal

Cite this article

Wroblewski, A. (2019). Paroles diffamantes, images infamantes. Captures, 4(1). https://doi.org/10.7202/1060152ar

\section{Article abstract}

La création artistique peut-elle véritablement faire du mal? Si oui, le mal, est-il tout de suite visible, palpable? Ou bien, le mal ne surgit-il que plus tard, après que le temps et l'histoire lui ont donné tort ou raison? Afin d'esquisser une éthique ou une esthétique de l'infamie et de la diffamation, ce numéro de Captures étudie des œuvres qui ont été dénoncées par le tribunal de l'opinion publique ou accusées et sanctionnées par la justice.
Tous droits réservés @ A Ania Wroblewski, 2019

\section{(c) (i) $\Theta$}

This document is protected by copyright law. Use of the services of Érudit (including reproduction) is subject to its terms and conditions, which can be viewed online.

https://apropos.erudit.org/en/users/policy-on-use/ 
Version enrichie de cet article : http://revuecaptures.org/node/3593

\section{Paroles diffamantes, images infamantes}

\section{Ania Wroblewski}

Résumé :

La création artistique peut-elle véritablement faire du mal? Si oui, le mal, est-il tout de suite visible, palpable? Ou bien, le mal ne surgit-il que plus tard, après que le temps et l'histoire lui ont donné tort ou raison? Afin d'esquisser une éthique ou une esthétique de l'infamie et de la diffamation, ce numéro de Captures étudie des œuvres qui ont été dénoncées par le tribunal de l'opinion publique ou accusées et sanctionnées par la justice.

Can art or literature do harm? If so, is the harm immediately visible and palpable? Or does the supposed harm done by creative works emerge later, after time and history have provided a context and means for their analysis? This issue of Captures explores how the concepts of libel and infamy bear on art production by examining works that have been denounced by the tribunal of public opinion or accused and sanctioned by the law.

Le sujet qui réunit les articles du dossier est celui de la diffamation et de l'infamie dans leurs déclinaisons les plus diverses, qu'elles soient artistiques, littéraires, cinématographiques, médiatiques ou autres. II est autant question d'œuvres dénoncées par le tribunal de l'opinion publique que d'ouvrages accusés ou sanctionnés par la justice. Bien qu'il n'existe aucune caractérisation reconnue des traits essentiels d'une œuvre dite diffamatoire ou infâme et que les artistes qu'on couvre d'infamie ou qu'on accuse de diffamation se complaisent parfois volontiers dans la gloire d'être détesté.e.s, les auteur.e.s du dossier nous indiquent la fonction de ces appellations : quand nous parlons de l'infamie et de la diffamation, nous signalons le fait qu'il y a eu une offense, nous pointons vers le tabou, nous mettons en évidence les enjeux éthiques, sociaux, historiques, institutionnels et juridiques de la représentation. Comme nous le rappelle Gisèle Sapiro dans son ouvrage La responsabilité de l'écrivain. Littérature, droit et morale en France (XIXe-XXIe siècle), "le scandale que produit une œuvre [...] constitue un bon révélateur des frontières du pensable et surtout du dicible ou du représentable dans une configuration socio-historique donnée » $(2011,35)$. Les articles de ce dossier nous aident à cerner les frontières évoquées par Sapiro, et ce, dans des contextes variés.

Dans leurs analyses, les auteur.e.s du dossier parlent de dommages collatéraux, de la dérive des médias, du rôle de l'État dans la promotion et la condamnation de certaines œuvres artistiques, de l'écart qui existe entre la vérité littéraire et la vérité juridique, de l'influence qu'exerce la production culturelle sur l'opinion publique. Elles et ils évoquent aussi - parfois en sourdine, d'autres fois, directement - l'idée de la mauvaise foi, autant celle des créatrices et des créateurs qui font prévaloir l'œuvre en devenir sur toute considération éthique et 
morale, autant celle des critiques qui, comme l'écrit Christophe Meurée dans son article du présent dossier, contournent parfois les éléments problématiques voire outrageux d'un ouvrage pour en souligner tout simplement la beauté. Meurée cite Jacques Marx quand il pose la question suivante : « La dimension esthétique d'une œuvre peut-elle camoufler son projet idéologique? » Comme nous le démontrent avec intelligence et rigueur les auteur.e.s du dossier, l'art, la littérature, le journalisme, le cinéma et la caricature existent dans un champ actif, animé, contemporain de réception. Provocatrices, compliquées et souvent hautement médiatisées, les œuvres dites diffamatoires ou infâmes ici étudiées n'entrent pas facilement dans le cadre des analyses sociohistoriques de l'art et de la littérature. Cependant, c'est précisément à cause du fait qu'elles mettent à l'épreuve les hiérarchies de valeurs des institutions culturelles qu'elles nous défient de les étudier sérieusement. Ces objets contestés soulèvent la question de l'éthique de la création artistique, font naître des discours sur la liberté d'expression et témoignent de la véritable force des arts dans le réel.

À cette fin, Eftihia Mihelakis s'appuie sur la pensée de Michel Foucault pour interroger la notion même de l'infamie qui se précise tout en se transformant à chaque fois qu'un.e écrivain.e trouble l'ordre bienséant du discours. Mihelakis conçoit l'écriture de la vie des autres par les auteures contemporaines de l'autofiction comme un geste de récidive. Christophe Meurée s'intéresse au cas de l'avocat bruxellois Edmond Picard, auteur d'œuvres à caractère antisémite qui jouissait d'une bonne réputation de son vivant - à l'époque de l'affaire Dreyfus. Meurée décèle dans la réception et la canonisation des œuvres antisémites de Picard les traces du populisme contemporain et constate que « juger de l'infamie, au regard de l'Histoire et de ses leçons, ne doit pas entraîner une suspicion et une révision anachronique des discours rangés sous cette étiquette ». Soline Asselin, quant à elle, aborde le sujet de la distance de plus en plus ténue entre l'art et le monde en dégageant de la foire médiatique provoquée par la publication en Norvège de Min Kamp de Karl Ove Knausgård les caractéristiques de ce que certains considèrent comme une « littérature de Judas ». Marie-Odile Richard et Louis-Daniel Godin entrent dans les interstices de l'affaire Grégory, une affaire criminelle française renouvelée régulièrement depuis 1984 et toujours en suspens, en s'intéressant à son appropriation littéraire et médiatique. Leurs analyses apportent un éclairage nouveau sur la tension - qui ne cesse de croître et que la fiction ne fait qu'exacerber - provoquée par l'atteinte présumée à la vie privée, un délit commis quand la représentation s'empare trop grossièrement ou sans merci de la réalité. Finalement, Maïté Snauwaert, spécialiste des théories du care et dont le projet intellectuel est celui d'identifier les facteurs littéraires conducteurs d'éthique, s'interroge sur la difficulté, de nos jours, de rendre compte de la mort d'un enfant en régime fictionnel.

Les contrepoints du numéro sont consacrés à des images qui, d'une façon ou d'une autre, abordent le caractère infamant de la représentation visuelle. Gina Cortopassi, Nancy Lemieux, Itay Sapir et Jean-Philippe Uzel nous présentent, respectivement, la vidéo The Key of $F(2018)$ de Andrea Cooper, les peintures réalisées 
en prison au cours des années 80 par le meurtrier en série John Wayne Gacy, les dessins préparatoires pour les Pittura infamante (1530) d'Andrea Del Sarto et la sculpture satirique Le Bity (1998) de l'artiste Olivier Blanckart. Kevin Lambert et Léonore Brassard signent des contrepoints littéraires sur Larry Clark et VALIE EXPORT, respectivement, qui rendent comptent de la puissance formidable que peuvent exercer sur nous les images qui nous touchent et qui nous troublent.

Mais la création artistique peut-elle véritablement faire du mal? Si oui, le mal, est-il tout de suite visible, palpable? Ou bien, le mal, tantôt symbolique, tantôt réel, ne surgit-il que plus tard, après que le temps et l'histoire lui ont donné tort ou raison? Trahison et fidélité, injure et éloge, insolence et discrétion, blasphème et respect, immoralité et moralité, impudeur et pudeur, scandale et instruction, humour et sérieux, mauvaise foi et bonne volonté : c'est autour de ces oppositions que s'élaborent les réflexions des auteur.e.s du dossier, auteur.e.s dont la tâche partagée est celle de théoriser une éthique ou bien une esthétique de l'infamie et de la diffamation.

\section{Bibliographie}

SAPIRO, Gisèle. 2011. La Responsabilité de l'écrivain. Littérature, droit et morale en France (XIXe-XXIe siècle). Paris : Seuil, $752 \mathrm{p}$. 